\section{Start typing TITLE here}

\section{Read instructions carefully before using this form.}

\title{
ABSTRACT FORM
}

IN STTU STUDIES OF FRACTURE IN SOLIDS." J. Narayan and S. M. Ohr. Solid State Division, Oak Ridge National Laboratory, Oak Ridge, Tennessee 37830

Electron microscope studies have been made of the propagation of microcracks during in situ tensile deformation of stainless steel, molybdenum and magnesium oxide representing ductile, scmi-brittle and brittle solids. As the stress is applied, the cracks are initiated at the edge of holes in the thinned foils. The extent of plastic activity around the cracks is measured in terms of the density and the distribution of dislocations and is sensitive to the ductile/brittle nature of the solid. The slip systems of the dislocations are determined by contrast analysis and stereoscopic observation. In stainless steel and magnesium oxide, the dislocations are relatively straight and they lie nearly perpendicular to the direction of the crack propagation, whereas in molybdenum the dislocations are in the form of irregular tangles which are stretched along the direction of the crack propagation. These observations are discussed in terms of the model of Dugdale concerning the formation of plastic zones under uniaxial loading in plane stress conditions. $\therefore$

"Research sponsored by the Department of Energy under contract with Union Carbide Corporation.

\section{DO NOT FOLD}

Signature of presenting author

Name of presenting author _._. . M. Ohr

Institution _._. Oak.Pidge National Laboratory

Full mailing address _. Solid_State Division, Oak_Ridge_National Laboratory P. 0. Box X, Oak Ridge, Tennessee 37830 


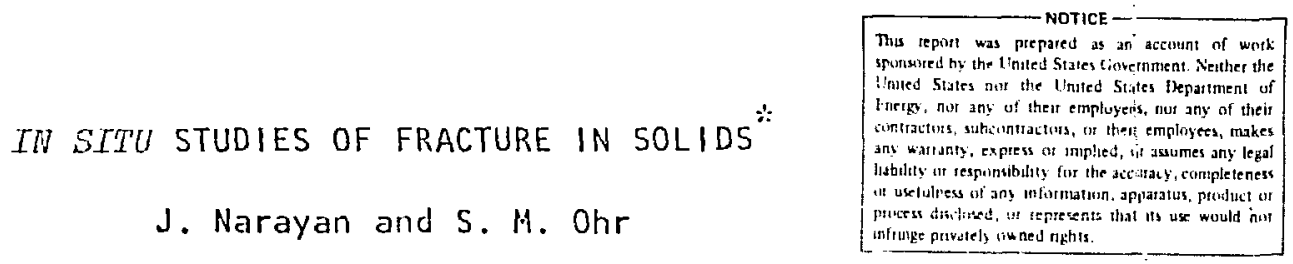

Solid State Division, Oak Ridge National Laboratory Oak Ridge, Tennesset, USA 37830

Problems of crack nucleation and propagation associated with fracture in solids have been the subject of numerous experimental and theoretical investigations in the past, but the basic mechanism of the phenomena is not well understood at present. One of the difficulties stems from the fact that detailed microscopic information on the physical processes occurring at the crack tip is lacking. This is particularly true with respect to the formation of plastic zones involving the generation and motion of dislocations. In the present study, we have examined by transmission electron microscopy the propagation of cracks during in situ tensile deformation of stainless steel, molybdenum and magnesium oxide representing ductile, semi-brittle and brittle solids.

Single crystal specimens suitable for electron transmission were prepared by dimpling and polishing from one side. With this geometry, the specimens could be deformed in pure tension in a bending holder. 1 As the stress was applied, the cracks were initiated at the edge of the holes in the thinned foils. The cracks were made to propagate into the crystal by gradually increasing the stress. The extent of plastic activity around the cracks was measured in terms of the density and the distribution of dislocations. The slip systems of the dislocations were determined by contrast analysis and stereoscopic observation.

Figure I shows a micrograph taken from a single crystal sample of type 316 stainless steel representing ductile solids. From the crystallographic analysis the fracture plane was identified as (111). The most noticeable feature is the band ahead of the crack tip with intense plastic activity. The dislocation density is extremely high near the crack tip, and it decreases gradually away from the tip. The band is made up of dislocations lying in the (111) plane which would have Burgers vectors a/2[101] and/or_a/2[0il]. Evidence of plastic activity on the other two slip planes, namely on ( $11 \overline{1}$ ) and (i 11$)$, can be seen as narrow slip bands running obliquenly into the crack plane. Figure 2 shows a similar observation made in a single crystal of molybdenum representing semi-brittle solids. It can be seen that the dislocations are in the form of irregular tangles which are stretched along the direction of the crack propagation. The crack plane was identified as the (110) plane and the Burgers vectors of the dislocations were a/2[111]] and/or a/2[11i]]. Figure 3 shows a crack observed in single crystal of Mgo representing brittle materials. The crack plane was identified as the (100) plane, and it ran along the [010] direction. The dislocations observed in the vicinity of the cracks are in contrast under the (200) reflection. These dislocations were in contrast under the $(220)$ and $(220)$ reflections but were out of contrast under the (020) reflection. From this contrast behavior, the Burgers vectors of these dislocations were deduced to be $a / 2[101]$ and $a / 2[101]$. From the stereomicroscopy and other geometric considerations, it appears that the dislocations on one side of the crack represent the $[101] /(101)$ slip system while those on the other side the $[101] /(101)$ slip system. 
Figure 1 shows a micrograph taken from a single crystal sample of type 316 stainless steel representing ductile solids. From the crystallographic analysis the fracture plane was identified as (i11). The most noticeable feature is the band ahead of the crack tip with intense plastic activity. The dislocation density is extremely high near the crack tip, and it decreases gradually away from the tip. The band is made up of dislocations lying in the (111) plane which would have Burgers vectors a/2[101] and/or a/2[0il]. Evidence of plastic activity on the other two slip planes, namely on (11i) and ( $1 \overline{1} 1)$, can be seen as narrow slip bands running obliquenly into the crack plane. Figure 2 shows a similar observation made in a single crystal of molybdenum representing semi-brittle solids. It can be seen that the dislocations are in the form of irregular tangles which are stretched along the direction of the crack propagation. The crack plane was identified as the (110) plane and the Burgers vectors of the dislocations were a/2[111] and/or a/2[111]. Figure 3 shows a crack observed in single crystal of Mgo representing brittle materials. The crack plane was identified as the (100) plane, and it ran along the [010] direction. The dislocations observed in the vicinity of the cracks are in contrast under the $(200)$ reflection. These dislocations were in contrast under the $(220)$ and $(2 \overline{2} 0)$ reflections but were out of contrast under the (020) reflection. From this contrast behavior, the Burgers vectors of these dislocations were deduced to be $a / 2[101]$ and $a / 2[101]$. From the stereomicroscopy and other geometric considerations, it appears that the dislocations on one side of the crack represent the $[101] /(101)$ slip system while those on the other side the $[101] /(101)$ slip system.

According to Dugdale, 2 the plastic zones under uniaxial tensile loading in plane stress should extend out on two $45^{\circ}$ planes which bisect the angle between the tensile axis and the leading edge of the crack and which are parallel to the direction of crack propagation. This type of behavior was observed by Hahn and Rosenfield 3 in their sheet tensile samples by the etch-pit technique. The present observations represent the electron microscope evidence for the formation of plastic zones near a crack similar to those expected from theory and thus render support for the validity of thin foil electron microscope techniques.

"Research sponsored by the Department of Energy under contract with Union Carbide Corporation. 
(1) T. S. Noggle and J. Narayan, Proc. 34th Ann. Meeting of EMSA, G. W. Bailey (ed.), 1976, p. 438.

(2) D. S. Dugdale, J. Mech. Phys. Solids 8, 100 (1960).

(3) G. T. Hahn and A. R. Rosenfield, Acta Met. 13, 293 (1965).

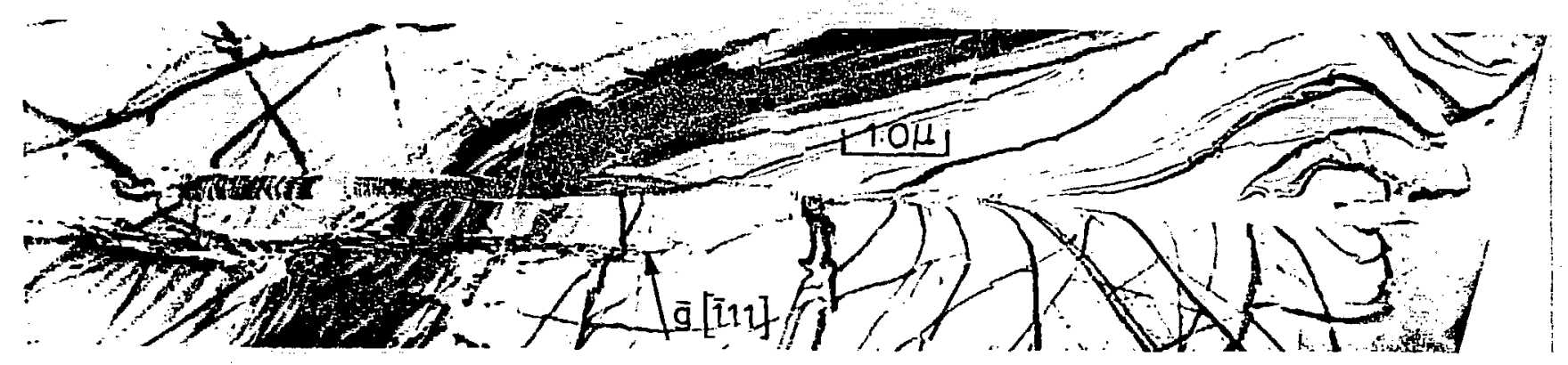

Fig. 1 Dislocation structure around a propagating crack in type 316 stainless steel single crystal sample. The sample was deformed in the electron microscope in pure tension.

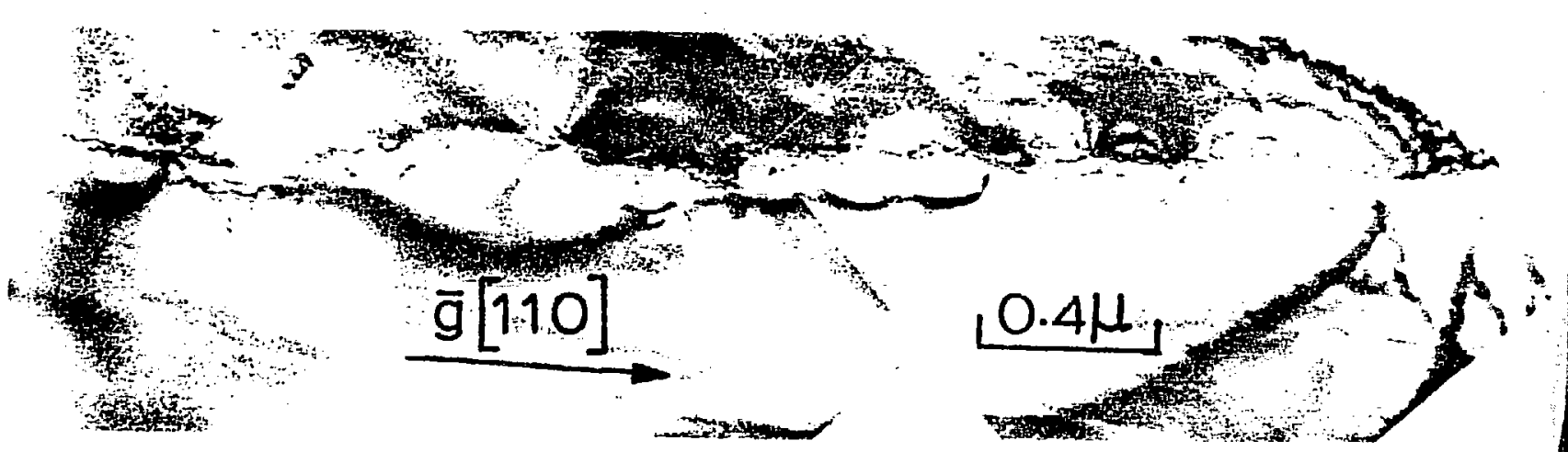

Fig. 2 Dislocation structure around a crack in molybdenum single crystal sample. The dislocations are in the form of irregular tangles which are stretched along the direction of the crack propagation. 


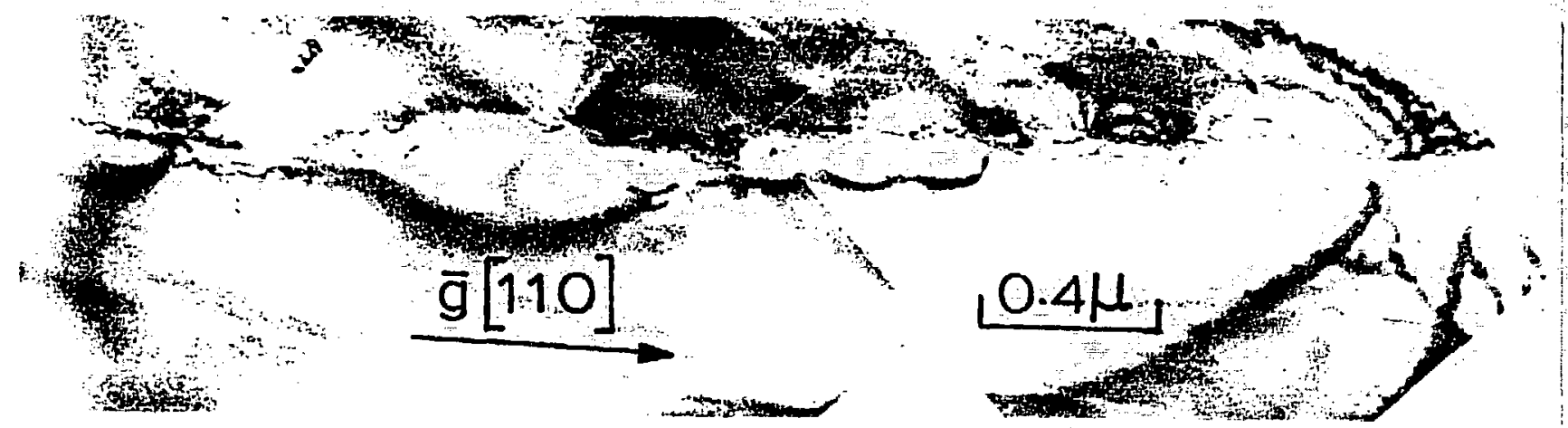

Fig. 2 Dislocation structure around a crack in molybdenum single crystal sample. The dislocations are in the form of irregular tangles which are stretched along the direction of the crack propagation.

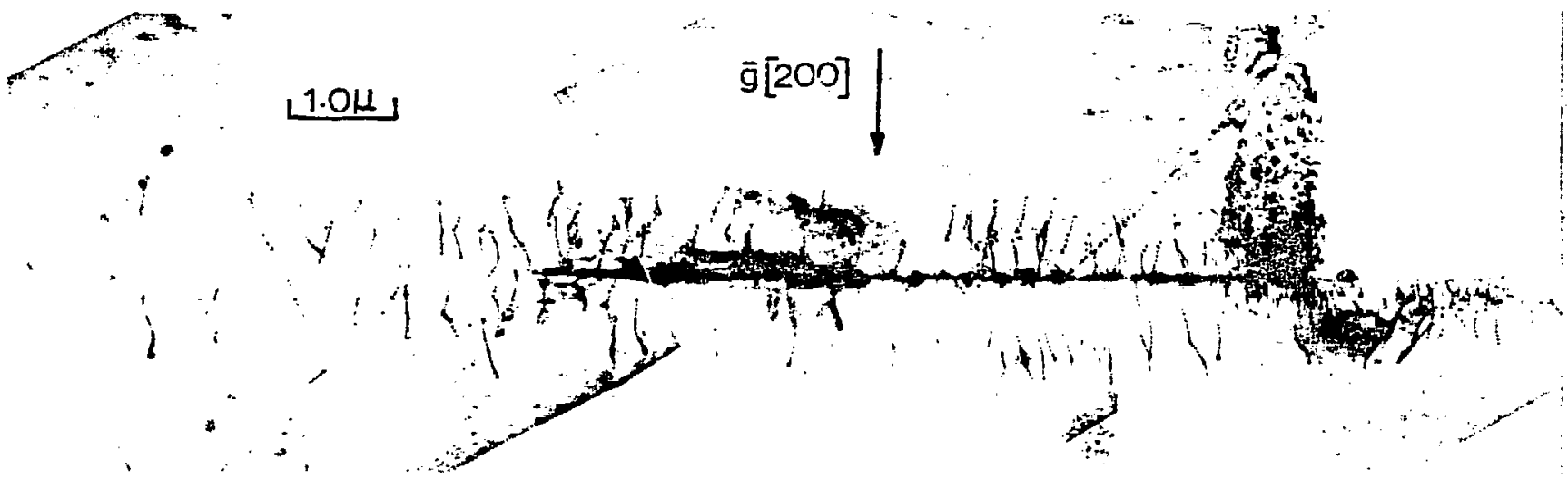

Fig. 3 Dislocation structure around a crack in an Mgo single crystal. The dislocations are associated with the $45^{\circ}$ shear-type plastic zones of the $[101] /(10 i)$ slip systems.

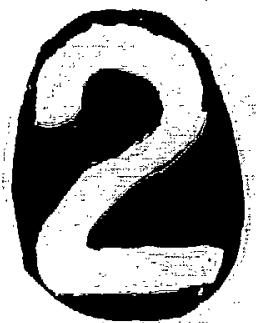

